\title{
LTL Generalized Model Checking Revisited
}

\author{
Patrice Godefroid $^{1}$, Nir Piterman ${ }^{2 \star}$ \\ 1 Microsoft Research \\ ${ }^{2}$ University of Leicester
}

Received: date / Revised version: date

\begin{abstract}
Given a 3-valued abstraction of a program (possibly generated using static program analysis and predicate abstraction) and a temporal logic formula, generalized model checking (GMC) checks whether there exists a concretization of that abstraction that satisfies the formula. In this paper, we revisit generalized model checking for linear time (LTL) properties. First, we show that LTL GMC is 2EXPTIMEcomplete in the size of the formula and polynomial in the model, where the degree of the polynomial depends on the formula, instead of EXPTIME-complete and quadratic as previously believed. The standard definition of GMC depends on a definition of concretization which is tailored for branchingtime model checking. We then study a simpler linear completeness preorder for relating program abstractions. We show that LTL GMC with this weaker preorder is only EXPSPACEcomplete in the size of the formula, and can be solved in linear time and logarithmic space in the size of the model. Finally, we identify classes of formulas for which the model complexity of standard GMC is reduced.
\end{abstract}

\section{Introduction}

Generalized model checking [BG00] is a way to improve precision when reasoning about partially defined systems. Such systems can be modeled as 3-valued Kripke structures where atomic propositions are either true, false or unknown, denoted by the third value $\perp$. Three-valued models are a natural representation of program abstractions generated automatically [GHJ01,GWC06] using static program analysis and predicate abstraction [GS97] for software model checking [BR01]. For an introduction to three-valued models we refer the reader to [BG99, GJ03, $\left.\mathrm{AHL}^{+} 08\right]$.

\footnotetext{
* Supported by the UK EPSRC project Complete and Efficient Checks for Branching-Time Abstractions.
}

Given a 3-valued model $M$ and a temporal-logic formula $\phi$, the generalized model-checking (GMC) problem is to decide whether there exists a complete system $M^{\prime}$ that is consistent with $M$ and that satisfies the formula $\phi$. From a practical point of view, generalized model checking can sometimes [GH05, GC05] improve verification of program abstractions. From a theoretical point of view, studying GMC is arguably interesting in its own right since GMC generalizes both model checking (when the values of all propositions in the model are known) and satisfiability checking (when all proposition values are unknown), probably the two most studied problems related to temporal logic and verification.

In this paper, we revisit GMC for linear-time temporallogic (LTL) formulas. We start by showing that LTL GMC is 2EXPTIME-complete in the size of the formula and polynomial in the model, where the degree of the polynomial depends on the formula, instead of EXPTIME-complete and quadratic as previously stated erroneously in [BG00]. The definition of GMC depends on the exact notion of abstraction, and is usually tailored for branching-time model checking [BG00]. We then study a simpler linear completeness preorder for relating program abstractions. We show that LTL GMC with this weaker preorder is only EXPSPACE-complete in the size of the formula, and can be solved in linear time and logarithmic space in the size of the model. Finally, we identify classes of formulas for which the model complexity of GMC defined with the standard branching-time completeness preorder is reduced.

Example 1. Consider the program $P$ :

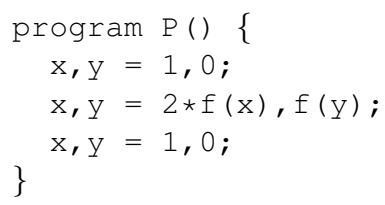

where $x$ and $y$ denote int variables, $f$ : int $->$ int denotes some unknown function, and the notation " $\mathrm{x}, \mathrm{y}=$ 1,0 " means variables $x$ and $y$ are simultaneously assigned 
values 1 and 0 , respectively. Let $\phi_{1}$ denote the LTL formula $F p_{y} \wedge G\left(p_{x} \vee \neg p_{y}\right)$ with the two predicates $p_{x}$ : "is x odd?" and $p_{y}$ : "is y odd?", and where $F$ means "eventually" while $G$ means "always", and let $\phi_{2}$ denote the LTL formula $X p_{y} \wedge$ $G\left(p_{x} \vee \neg p_{y}\right)$, where $X$ means"next" (see the next section for formal definitions).

Given such a program and knowing the predicate of interests $p_{x}$ and $p_{y}$, predicate abstraction can be used to automatically generate the following 3-valued Kripke structure $M$ (or "Boolean program" [BR01]) abstracting $P$ [GHJ01]:

$$
\begin{array}{lll}
\text { initial state } s_{0}: & p_{x}=\text { true }, & p_{y}=\text { false } \\
\text { next state } s_{1}: & p_{x}=\text { false }, & p_{y}=\perp \\
\text { next state } s_{2}: & p_{x}=\text { true }, & p_{y}=\text { false }
\end{array}
$$

loop forever in $s_{2}$

As shown in [GJ02] and discussed later, model checking ${ }^{1} \phi_{1}$ and $\phi_{2}$ against $M$ returns the value "unknown," while generalized model checking can prove that no concretization of $M$ can possibly satisfy either $\phi_{1}$ or $\phi_{2}$, i.e., no matter how function $\mathrm{f}$ is implemented.

Although $\phi_{2}=X p_{y} \wedge G\left(p_{x} \vee \neg p_{y}\right)$ is an LTL safety formula and hence is within the scope of predicate-abstractionbased software model checkers such as SLAM [BR01] or BLAST [HJMS02], these tools cannot prove that $\phi_{2}$ does not hold regardless of the definition of function $f$ : this result can only be obtained through generalized model checking. Instead, when confronted with such a program $P$, these tools would attempt to iteratively refine the abstraction $M$ by analyzing the code of function $f$ if it is available. This process is in general exponential in the size of the abstraction, since adding a single predicate in each iteration may double the size of the abstraction. Moreover, this process may not terminate. For the above abstraction $M$ and formula $\phi_{2}$, the expensive and unpredictable abstraction-refinement process can thus be avoided thanks to GMC. Although the worst-case complexity of GMC is expensive in the size of the (usually short) formula (but so is traditional LTL model checking which is already PSPACE-complete), GMC can always be done in time polynomial in the size of the model (linear or quadratic in many cases as shown later), in contrast with abstraction refinement which is typically exponential in the (usually large) model.

\section{Preliminaries}

A partial Kripke structure (PKS for short) [BG99] is $M=$ $\left\langle S, R, L, s^{i n}\right\rangle$ where $S$ is a nonempty set of states, $R \subseteq S \times S$ is a total image-finite transition relation (i.e., every state has a non-zero finite number of immediate successor states), $L$ : $S \times A P \rightarrow \mathbf{3}$ is a labeling of states that associates a truth value in $\mathbf{3}=\{$ true,$\perp$, false $\}$ to each atomic proposition in a finite set $A P$, and $s^{i n} \in S$ is an initial state. For a state $s$ and proposition $p$, we say that $p$ is true in $s$ if $L(s, p)=$ true, it is false in $s$ if $L(s, p)=$ false, and it is unknown $\perp$ otherwise.

\footnotetext{
1 In model checking, we mean normal 3-valued model checking in the sense of [BG99].
}

A PKS is complete if the range of $L$ is $\mathbf{2}=\{$ true, false $\}$. We call a complete PKS a Kripke Structure or KS. When we want to stress that a PKS $M$ is complete, we denote it by $\bar{M}$. Given a state $s$, we denote by $L(s)$ the function $\sigma: A P \rightarrow \mathbf{3}$ such that $\sigma(p)=L(s, p)$. We use the notations $\mathbf{3}^{A P}=\{\sigma$ : $A P \rightarrow \mathbf{3}\}$ and $\mathbf{2}^{A P}=\{\sigma: A P \rightarrow \mathbf{2}\}$. For $s \in S$, we denote by $(M, s)$ the PKS $\langle S, R, L, s\rangle$.

A computation from $s_{0} \in S$ is $s_{0}, s_{1}, \ldots$ such that forall $i \geq 0$ we have $\left(s_{i}, s_{i+1}\right) \in R$. A computation $\pi=s_{0}, s_{1}, \ldots$ induces a trace $L(\pi)=L\left(s_{0}\right) L\left(s_{1}\right) \cdots \in\left(3^{A P}\right)^{\omega}$. A computation $\pi$ is called a computation of $M$ if $s_{0}=s^{i n}$. The set of computations of $M$ that start in $s$ is denoted $\mathcal{C}(M, s)$ and the set of induced traces is denoted $\mathcal{L}(M, s)$. We abbreviate $\mathcal{C}\left(M, s^{i n}\right)$ as $\mathcal{C}(M)$ and $\mathcal{L}\left(M, s^{i n}\right)$ as $\mathcal{L}(M)$. In general, $\mathcal{L}(M, s) \subseteq\left(3^{A P}\right)^{\omega}$. Given a PKS $M=\left\langle S, R, L, s^{i n}\right\rangle$, the unwinding of $M$ into a tree is the PKS $M^{+}=\left\langle S^{+}, R^{\prime}, L^{\prime}, s^{i n}\right\rangle$, where $S^{+}$is the set of nonempty sequences over $S, R^{\prime}=$ $\left\{\left(s_{1} \cdots s_{n}, s_{1} \cdots s_{n} \cdot s_{n+1}\right) \in\left(S^{+} \times S^{+}\right) \mid\left(s_{n}, s_{n+1}\right) \in R\right\}$, and $L^{\prime}(\pi \cdot s)=L(s)$. We restrict the set $S^{+}$to the set of sequences reachable from $s^{i n}$. If $M$ is a Kripke structure then so is $M^{+}$.

To interpret temporal logic formulas on PKSs, we extend Kleene's strong 3-valued propositional logic [Kle87]. Conjunction $\wedge$ in this logic is defined as the minimum $M$ in of its arguments with respect to the truth ordering $\leq_{T}$ where false $\leq_{T} \perp \leq_{T}$ true. We extend this function to sets in the obvious way, with $\operatorname{Min}(\emptyset)=$ true. Negation $\neg$ is defined using the function 'Comp' that maps true to false, false to true, and $\perp$ to $\perp$. Disjunction $\vee$ is defined as usual using De Morgan's laws: $p \vee q=\neg(\neg p \wedge \neg q)$. Propositional modal logic (PML) is propositional logic extended with the modal operator $A X$ (which is read "for all immediate successors"). Formulas of PML have the following abstract syntax:

$$
\phi::=p|\neg \phi| \phi_{1} \wedge \phi_{2} \mid A X \phi,
$$

where $p$ ranges over $A P$. The following 3-valued semantics generalizes the traditional 2-valued semantics for PML.

Definition 1. The value of a formula $\phi$ of 3-valued PML in a state $s$ of a PKS $M=\left\langle S, R, L, s^{i n}\right\rangle$, written $[(M, s) \models \phi]$, is defined inductively as follows:

$$
\begin{aligned}
{[(M, s) \models p] } & =L(s, p) \\
{[(M, s) \models \neg \phi] } & =\operatorname{Comp}([(M, s) \models \phi]) \\
{\left[(M, s) \models \phi_{1} \wedge \phi_{2}\right] } & =\operatorname{Min}\left(\left\{\left[(M, s) \models \phi_{i}\right] \mid i \in\{1,2\}\right\}\right) \\
{[(M, s) \models A X \phi] } & =\operatorname{Min}\left(\left\{\left[\left(M, s^{\prime}\right) \models \phi\right] \mid\left(s, s^{\prime}\right) \in R\right\}\right)
\end{aligned}
$$

We write $[M \models \phi]$ for $\left[\left(M, s^{i n}\right) \models \phi\right]$. This 3-valued logic can be used to define a preorder $\preceq$ on PKSs that reflects their degree of completeness. Let $\leq_{I}$ be the information ordering on truth values where $\perp$ is the least element and true and false are maximal uncomparable elements: $\perp \leq_{I}$ true, false. For two PKS $M_{i}=\left\langle S_{i}, R_{i}, L_{i}, s_{i}^{i n}\right\rangle$ with $i=1,2$ the completeness preorder is the greatest relation $\preceq \subseteq S_{1} \times S_{2}$ such that $s_{1} \preceq s_{2}$ implies all the following:

1. For every $p \in A P$, we have $L_{1}\left(s_{1}, p\right) \leq_{I} L_{2}\left(s_{2}, p\right)$.

2. For every $\left(s_{1}, s_{1}^{\prime}\right) \in R_{1}$, there exists $\left(s_{2}, s_{2}^{\prime}\right) \in R_{2}$ such that $s_{1}^{\prime} \preceq s_{2}^{\prime}$. 
3. For every $\left(s_{2}, s_{2}^{\prime}\right) \in R_{2}$, there exists $\left(s_{1}, s_{1}^{\prime}\right) \in R_{1}$ such that $s_{1}^{\prime} \preceq s_{2}^{\prime}$.

We say that $M_{2}$ is more complete than $M_{1}$, denoted $M_{1} \preceq$ $M_{2}$, if $s_{1}^{i n} \preceq s_{2}^{i n}$. It can be shown that 3-valued PML logically characterizes the completeness preorder.

Theorem 1. [BG99] Let $M_{1}$ and $M_{2}$ be partial Kripke structures, and let $\Phi$ be the set of all formulas of 3-valued $P M L$. Then $M_{1} \preceq M_{2}$ iff $\left(\forall \phi \in \Phi:\left[M_{1} \models \phi\right] \leq_{I}\left[M_{2} \models \phi\right]\right)$.

In other words, partial Kripke structures that are "more complete" with respect to $\preceq$ have more definite properties with respect to $\leq_{I}$, i.e., have more properties that can be established true or false by model checking. Moreover, every formula $\phi$ of 3-valued PML that evaluates to true or false on a partial Kripke structure has the same truth value when evaluated on a more complete structure.

\subsection{Model Checking and Generalized Model Checking}

The sets of LTL and CTL formulas are defined as follows.

$$
\begin{gathered}
\operatorname{LTL} \varphi::=p|\varphi \wedge \varphi| \neg \varphi|X \varphi| \varphi U \varphi \\
\operatorname{CTL} \varphi::=p|\varphi \wedge \varphi| \neg \varphi|A X \varphi| A \varphi U \varphi \mid E \varphi U \varphi
\end{gathered}
$$

We assume familiarity with the semantics of LTL and CTL and with their model checking. As usual, we denote true $U \varphi$ by $F \varphi, \neg F \neg \varphi$ by $G \varphi$ and $\neg((\neg \varphi) U(\neg \psi))$ by $\varphi R \psi$. The above grammar includes a complete set of operators and other operators can be expressed in the usual way. Given a set of propositions $A P$ and an LTL formula $\varphi$, the language of $\varphi$, denoted $L(\varphi)$ is the set of models of $\varphi$ in $\left(2^{A P}\right)^{\omega}$. Formally, $L(\varphi)=\left\{w \in\left(\mathbf{2}^{A P}\right)^{\omega} \mid w \models \varphi\right\}$. The 3-valued semantics of LTL and CTL path formulas extend Definition 1 as expected.

Precisely, given a 3-valued infinite word $w=a_{0} a_{1} a_{2} \ldots \in$ $\left(3^{A P}\right)^{\omega}$, the 3 -valued semantics of the next LTL operator is defined as

$$
[w \models X \varphi]=\left[w^{\prime} \models \varphi\right]
$$

with $w^{\prime}=a_{1} a_{2} \cdots \in\left(\mathbf{3}^{A P}\right)^{\omega}$, while the 3 -valued semantics of the strong until LTL operator $U$ is defined as

$$
\left[w \models \varphi_{1} U \varphi_{2}\right]=
$$

$\operatorname{Max}\left(\left\{\operatorname{Min}\left(\left\{\left[a_{i} \models \varphi_{1}\right] \mid i<k\right\} \cup\left\{\left[a_{k} \mid=\varphi_{2}\right]\right\}\right) \mid k \geq 0\right\}\right)$

For a partial Kripke structure $M$ and a CTL formula $\phi$, we denote the value of $\phi$ at state $s$ by $[(M, s) \models \phi] \in \mathbf{3}^{A P}$. Let $\operatorname{Path}(s)$ denote all 3 -valued infinite paths starting at $s$. The 3-valued semantics of the CTL operators $A U$ and $E U$ are then defined as

$$
\begin{aligned}
{[(M, s) \models} & \left.A \varphi_{1} U \varphi_{2}\right]= \\
& \operatorname{Min}\left(\left\{\left[w \models \varphi_{1} U \varphi_{2}\right] \mid w \in \operatorname{Path}(s)\right\}\right)
\end{aligned}
$$

and as

$$
\begin{aligned}
{[(M, s) \models} & \left.E \varphi_{1} U \varphi_{2}\right]= \\
& \operatorname{Max}\left(\left\{\left[w \mid=\varphi_{1} U \varphi_{2}\right] \mid w \in \operatorname{Path}(s)\right\}\right)
\end{aligned}
$$

For the initial state $s^{i n}$ of $M$ we denote $\left[\left(M, s^{i n}\right) \models \phi\right]$ by $[M \models \phi]$. If $M$ is a Kripke structure we simply write $M, s \models \varphi$ for $[(M, s) \models \varphi]=$ true and $M, s \forall \varphi$ for $[(M, s) \models \varphi]=$ false. For a Kripke structure $\bar{M}$ and an LTL formula $\varphi$, we say that $\bar{M}$ satisfies $\varphi$, denoted $\bar{M}=\varphi$ if $\mathcal{L}(\bar{M}) \subseteq L(\varphi)$.

In practice, the size of the Kripke structure $\bar{M}$ can be prohibitively expensive or even infinite. Instead, a smaller (finite) abstraction $M^{\prime}$ can be used: if $M^{\prime}$ is generated in such a way that $M^{\prime} \preceq \bar{M}$, then all the properties $\phi$ that can be proved (true) or disproved (false) on $M^{\prime}$ will also hold on $\bar{M}$, by Theorem 1 . With static program analysis and predicate abstraction, generating such abstractions with respect to the completeness preorder $\preceq$ can be done at the same computational cost as computing standard abstractions that merely simulate (over-approximate) the concrete system $\bar{M}$ [GHJ01]. Moreover, 3-valued model checking can itself be done at the same computational cost as regular 2-valued model checking [BG00].

In some cases, precisely characterized in [GH05] and also independently studied in [GC05], all the completions of an abstraction $M$ agree on the satisfaction of a formula $\varphi$, yet 3valued model checking is not accurate enough to identify this and still returns $\perp$. For instance, this is the case for the formula $p \vee \neg p$ if $p$ is $\perp$. This observation suggests a more precise version of 3-valued model checking [BG00]: the value of a formula $\varphi$ in a PKS $M$ should be unknown only if some completions of $M$ satisfy $\varphi$ and some completions of $M$ falsify $\varphi$ [BG00]. We denote the value of $\varphi$ on $M$ according to this thorough semantics by $[M \models \varphi]_{t} \in \mathbf{3}$. Formally, we have the following.

$$
\begin{array}{ll}
{[M \mid=\varphi]_{t}=\text { true }} & \text { If } \forall \overline{M^{\prime}} \cdot M \preceq \overline{M^{\prime}} \rightarrow \overline{M^{\prime}} \models \varphi \\
{[M \mid=\varphi]_{t}=\text { false }} & \text { If } \forall \overline{M^{\prime}} \cdot M \preceq \overline{M^{\prime}} \rightarrow \bar{M}^{\prime} \not \varphi \varphi \\
{[M \mid=\varphi]_{t}=\perp} & \text { If } \exists \bar{M}_{1}, \bar{M}_{2} \cdot M \preceq \bar{M}_{1}, M \preceq \bar{M}_{2}, \\
& \bar{M}_{1} \models \varphi, \text { and } \bar{M}_{2} \not \models \varphi
\end{array}
$$

Generalized model checking (GMC) can determine the value of $[M \models \varphi]_{t}$ [BG00]. Given a PKS $M$ and a formula $\varphi$, the GMC problem for $M$ and $\varphi$ is to determine whether there exists a Kripke structure $M^{\prime}$ that completes $M$ and satisfies $\varphi$. Formally, we have the following.

$$
M \models \preceq \varphi \text { iff there exists } \overline{M^{\prime}} \succeq M \text { such that } \overline{M^{\prime}} \models \varphi
$$

The value $[M \models \varphi]_{t}$ can be evaluated with two GMC questions. First, we check whether $M \models \preceq \varphi$. If the answer is no, then all completions of $M$ do not satisfy $\varphi$ and $[M \models$ $\varphi]_{t}=$ false. If the answer is yes, we next check whether $M \mid \_\neg \varphi$. If that answer is no, then we know that all completions of $M$ satisfy $\varphi$ and $[M \models \varphi]_{t}=$ true. Otherwise, $[M \models \varphi]_{t}=\perp$.

It can be shown that 3-valued model checking is sound with respect to the thorough semantics.

Theorem 2. [BG00] Let $M$ be a PKS and $\varphi$ an LTL or CTL formula.

1. $[M \models \varphi]=$ true implies $[M \models \varphi]_{t}=$ true. 
2. $[M \models \varphi]=$ false implies $[M \models \varphi]_{t}=$ false.

In this paper we revisit LTL generalized model checking and show that its complexity is greater than what was previously believed. We also consider specifications (both in LTL and CTL) for which the model complexity of generalized model checking is simpler than the general case.

\subsection{Automata over Infinite Words}

We assume familiarity with the basic notions of alternating automata on infinite objects, cf. [GTW02]. We formally define automata on infinite words and refer to automata on infinite trees without a formal proof.

For an alphabet $\Sigma$, the set $\Sigma^{*}$ is the set of finite sequences of elements from $\Sigma$. For $x \in \Sigma^{*}$, we denote the length of $x$ by $|x|$. Given an alphabet $\Sigma$ and a set $D$ of directions, a $\Sigma$ labeled $D$-tree is a pair $\langle T, \tau\rangle$, where $T \subseteq D^{*}$ is a tree over $D$ and $\tau: T \rightarrow \Sigma$ maps each node of $T$ to a letter in $\Sigma$. A path $\pi$ of a tree $T$ is a set $\pi \subseteq T$ such that $\epsilon \in \pi$ and for every $x \in \pi$ either $x$ is a leaf in $T$ or there exists a unique $\gamma \in D$ such that $x \cdot \gamma \in \pi$. For $\pi=\gamma_{1} \cdot \gamma_{2} \cdots$, we write $\tau(\pi)$ for $\tau(\epsilon) \cdot \tau\left(\gamma_{1}\right) \cdot \tau\left(\gamma_{1} \gamma_{2}\right) \cdots$.

For a finite set $X$, let $\mathcal{B}^{+}(X)$ be the set of positive Boolean formulas over $X$ (i.e., Boolean formulas built from elements in $X$ using $\wedge$ and $\vee$ ), where we also allow the formulas true and false. For a set $Y \subseteq X$ and a formula $\theta \in \mathcal{B}^{+}(X)$, we say that $Y$ satisfies $\theta$ iff assigning true to elements in $Y$ and assigning false to elements in $X \backslash Y$ makes $\theta$ true. An alternating word automaton is $A=\left\langle\Sigma, Q, q_{i n}, \delta, \alpha\right\rangle$, where $\Sigma$ is the input alphabet, $Q$ is a finite set of states, $\delta: Q \times \Sigma \rightarrow \mathcal{B}^{+}(Q)$ is a transition function, $q_{i n} \in Q$ is an initial state, and $\alpha$ specifies the acceptance condition. A run of $A$ on $w=\sigma_{0} \sigma_{1} \cdots$ is a $Q$-labeled $D$-tree, $\langle T, \tau\rangle$, where $\tau(\epsilon)=q_{\text {in }}$ and, for every $x \in T$, we have $\left\{\tau\left(x \cdot \gamma_{1}\right), \ldots, \tau\left(x \cdot \gamma_{k}\right)\right\} \models \delta\left(\tau(x), \sigma_{|x|}\right)$ where $\left\{x \cdot \gamma_{1}, \ldots, x \cdot \gamma_{k}\right\}$ is the set of children of $x$. A run of $A$ is accepting if all its infinite paths satisfy the acceptance condition. For a path $\pi$, we denote the set of automaton states visited infinitely often along this path by in $f(\pi)$, i.e., inf $f(\pi)=\{q \mid q$ appears infinitely often in $\tau(\pi)\}$. We consider the following three acceptance conditions:

- A path $\pi$ satisfies a Büchi condition $\alpha \subseteq Q$ iff $\inf (\pi) \cap$ $\alpha \neq \emptyset$.

- A path $\pi$ satisfies a co-Büchi condition $\alpha \subseteq Q$ iff inf $(\pi) \cap$ $\alpha=\emptyset$.

- A path $\pi$ satisfies a parity condition $\alpha=\left\langle F_{0}, \ldots, F_{k}\right\rangle$ where $F_{0}, \ldots F_{k}$ form a partition of $Q$ iff for some even $i$ we have $\inf (\pi) \cap F_{i} \neq \emptyset$ and forall $i^{\prime}<i$ we have in $f(\pi) \cap F_{i^{\prime}}=\emptyset$. We call $k$ the number of priorities of $\alpha$.

For the three conditions, an automaton accepts a word iff there exists a run that accepts it. We denote by $\mathcal{L}(\mathcal{A})$ the set of all $\Sigma$-words that $A$ accepts.

Below we discuss some special cases of alternating automata. The alternating automaton $A$ is nondeterministic if for all the formulas that appear in $\delta$ are disjunctions over the states $Q$. The automaton $A$ is deterministic if all formulas that appear in $\delta$ are states from $Q$. For a nondeterministic automaton we write $\delta: Q \times \Sigma \rightarrow 2^{Q}$ and for a deterministic automaton we write $\delta: Q \times \Sigma \rightarrow Q$.

We denote each of the different types of automata by an acronym in $\{D, N, A\} \times\{W, B, C, P\} \times\{W, T\}$, where the first letter describes the branching mode of the automaton (deterministic, nondeterministic, or alternating), the second letter describes the acceptance condition (Weak, ${ }^{2}$ Büchi, coBüchi, or parity), and the third letter describes the object over which the automaton runs (words or trees). For example, an $\mathrm{ABW}$ is an alternating Büchi word automata and a DPW is a deterministic parity word automata.

We state the following well known results about automata and their relation to LTL.

Theorem 3. [VW94] For every LTL formula $\varphi$ of length $n$ there exist an $N B W N_{\varphi}$ with $2^{O(n)}$ states such that $L\left(N_{\varphi}\right)=$ $L(\varphi)$.

Theorem 4. [Saf88, Pit07] For every $N B W N$ with $m$ states there exists a DPW D with $O\left((m !)^{2}\right)$ states and $2 m$ priorities such that $L(N)=L(D)$.

Corollary 1. For every LTL formula $\varphi$ of length $n$ there exists a DPW $D_{\varphi}$ with $2^{2^{O(n \operatorname{logn})}}$ states and $2^{O(n)}$ priorities such that $L\left(D_{\varphi}\right)=L(\varphi)$.

Theorem 5. [Jur00] Given an APW A over a 1-letter alphabet with $n$ states and $k$ priorities, we can decide whether $L(A)=\emptyset$ in time $n^{O(k)}$.

Theorem 6. [SVW87] Given two $N B W N_{1}, N_{2}$ we can decide whether $L\left(N_{1}\right) \subseteq L\left(N_{2}\right)$ in space logarithmic in $N_{1}$ and polynomial in $N_{2}$.

\section{LTL Generalized Model Checking}

We show that, contrary to previous beliefs, GMC with respect to linear time logic is 2EXPTIME-complete. Our upper bound combines a DPW for the LTL property with the PKS to get an APW over a 1-letter alphabet. The APW is not empty iff the GMC problem holds. For the lower bound, we show a reduction from LTL realizability to generalized model checking. LTL realizability is 2EXPTIME-hard [PR89] establishing 2EXPTIME-hardness of generalized model checking. The two together establish 2EXPTIME-completeness of generalized model checking for LTL.

Theorem 7. LTL generalized model checking $M \models \preceq \varphi$ can be solved in polynomial time in the size of $M$ and double exponential time in the size of $\varphi$.

Proof. Consider an LTL formula $\varphi$. Let $|\varphi|=n$. According to Corollary 1 there exists a DPW $D_{\varphi}$ with $2^{2^{O(n \log n)}}$ states and $2^{O(n)}$ priorities such that $L(\varphi)=L\left(D_{\varphi}\right)$.

Let $D_{\varphi}=\left\langle\mathbf{2}^{A P}, Q, q_{0}, \rho, \alpha\right\rangle$ and $M=\left\langle S, R, L, s^{i n}\right\rangle$. Consider the following APW $A$ over a 1-letter alphabet that

\footnotetext{
2 We delay the definition of weak automata to Section 5.
} 
is obtained from the combination of $M$ and $D_{\varphi}$. We define $A=\left\langle\{a\}, Q \times S,\left(q_{0}, s^{i n}\right), \eta, \alpha^{\prime}\right\rangle$ such that

$$
\eta((q, s), a)=\bigvee_{\bar{\sigma} \succeq L(s)} \bigwedge_{\left(s, s^{\prime}\right) \in R}\left(\rho(q, \bar{\sigma}), s^{\prime}\right)
$$

and $\alpha^{\prime}=\left\langle F_{0}^{\prime}, \ldots, F_{k}^{\prime}\right\rangle$ is obtained from $\alpha=\left\langle F_{0}, \ldots, F_{k}\right\rangle$ by setting $F_{j}^{\prime}=F_{j} \times S$.

Lemma 1. A accepts $a^{\omega}$ iff $M \models \preceq \varphi$.

Proof. Suppose that $A$ accepts $a^{\omega}$. Let $\langle T, \tau\rangle$ be an accepting run of $A$ on $a^{\omega}$. Consider the following Kripke structure $\left\langle T, R^{\prime}, L\right\rangle$ where $R^{\prime}(x, x a)$ for every $x, x a \in T$ and $L: T \rightarrow \mathbf{2}^{A P}$ is defined as follows. Consider a node $x$ where $\tau(x)=(q, s)$ and $\left\{x a_{1}, \ldots, x a_{k}\right\}$ is the set of children of $x$. By definition of a run of $A$, there is a function $\sigma: A P \rightarrow\{$ true, false $\}$ such that $\left\{\tau\left(x a_{1}\right), \ldots, \tau\left(x a_{k}\right)\right\} \models$ $\bigwedge_{\left(s, s^{\prime}\right) \in R}\left(\rho(q, \sigma), s^{\prime}\right)$. We define $L(x)$ to be this function $\sigma$. It is easy to see that $\left\langle T, R^{\prime}, L\right\rangle$ is a Kripke structure that is more complete than $M$. Moreover, for every path $\pi$ in $\left\langle T, R^{\prime}, L\right\rangle$, the projection of $\tau$ on the states of $D_{\varphi}$ for this path defines an accepting run of $D_{\varphi}$ and implies that $L(\pi) \models \varphi$.

Conversely, suppose that there exists a Kripke structure $K=\left\langle S, R, L, s_{0}\right\rangle$ such that $M \preceq K$ and $K \models \varphi$. Consider the unwinding $K^{+}=\left\langle S^{+}, R^{\prime}, L^{\prime}, s_{0}\right\rangle$ of $K$. We construct a labeling $\tau: S^{+} \rightarrow Q \times S$ such that for every node $x=s_{0} s_{1} \cdots s_{n}$ we have $\tau(x)$ associates $x$ to a state $s$ of $M$ such that $s \preceq s_{n}$ and to a state $q$ of $D_{\varphi}$ such that $q=$ $\rho\left(q_{0}, L\left(s_{0} \cdots s_{n-1}\right)\right)$. Note that $q$ is unique for a given sequence $s_{0} \cdots s_{n-1}$ as the automaton $D_{\varphi}$ is deterministic. Initially, $\tau\left(s_{0}\right)=\left(q_{0}, s^{i n}\right)$. Obviously, $s^{i n} \preceq s_{0}$. Consider a node $x=s_{0} s_{1} \cdots s_{n}$ such that $\tau(x)=(q, s)$. Let $x s_{n+1}$ be a child of $x$. Then $\tau\left(x s_{n+1}\right)=\left(q^{\prime}, s^{\prime}\right)$ where $q^{\prime}=\rho\left(q, L\left(s_{n}\right)\right)$ and as $s \preceq s_{n}$ there exists a successor $s^{\prime}$ of $s$ such that $s^{\prime} \preceq s_{n+1}$. It is easy to see that $\left\langle S^{+}, \tau\right\rangle$ is a run tree of $A$ on $a^{\omega}$. By assumption every trace of $K$ is accepted by $D_{\varphi}$. It follows that every infinite path in $\left\langle S^{+}, \tau\right\rangle$ is labeled by an accepting run of $D_{\varphi}$ and that $\left\langle S^{+}, \tau\right\rangle$ is accepting.

According to Theorem 5 the emptiness of $A$ can be determined in time proportional to $\left(2^{2^{O(n \log n)}}\right)^{2^{O(n)}}=2^{2^{O(n \log n)}}$

Note that, if $D_{\varphi}$ was nondeterministic in the previous proof, it could not precisely track simultaneously different matching states $s$ such that $s \preceq s_{n}$ in the proof, and therefore $M \models_{\preceq} \varphi$ would not necessarily imply that $A$ accepts $a^{\omega}$. This is in essence the error in the proof of Theorem 25 of [BG00], which led to the overly optimistic EXPTIME upperbound.

Example 2. Consider the partial Kripke structure $M$ in Figure 1. The structure $M$ is an extended version of the program depicted in Example 1. Consider the formula $\varphi=F\left(\neg p_{x} \wedge\right.$ $\left.p_{y}\right) \vee G\left(\neg p_{y}\right)$. There is a concretization of $M$ that satisfies $\varphi$. Indeed, if we set $p_{y}=$ true in state $s_{2}$, then both traces of $M$ satisfy $\varphi$. Denote this concretization by $K_{t}$. Suppose that $D$ is an automaton for $\varphi$. Our algorithm uses $D$ to construct an APW over 1-letter alphabet that guesses the labels of $K_{t}$ and runs $D$ simultaneously on all traces of $K_{t}$. We demanded that $D$ be a deterministic automaton.

Consider the NBW $N_{\varphi}$ for $\varphi$ depicted in Figure 1. The NBW $N_{\varphi}$ is not strong enough to notice that $K_{t}$ satisfies $\varphi$. A composition of $N_{\varphi}$ and $M$ would start in state $\left(s_{0}, q_{0}\right)$. Then, $N_{\varphi}$ reads the initial state $p_{x} \wedge \neg p_{y}$ of $K_{t}$ and it has to guess whether to go to state $q_{1}$ or $q_{2}$. That is, whether the APW will move to state $\left(s_{1}, q_{1}\right)$ or $\left(s_{1}, q_{2}\right)$. However, there is no run of $N_{\varphi}$ starting from $q_{1}$ that accepts the path $s_{1}, s_{2}, s_{3}, \ldots$ and there is no run of $N_{\varphi}$ starting from $q_{2}$ that accepts the path $s_{1}, s_{4}, s_{5}, \ldots$. Effectively, $N_{\varphi}$ has to choose whether all paths of $K_{t}$ satisfy $G\left(\neg p_{y}\right)$ (by choosing the transition to state $q_{1}$ ) or all paths of $K_{t}$ satisfy $F\left(\neg p_{x} \wedge p_{y}\right)$ (by choosing the transition to state $q_{2}$ ). It follows that the language of an APW that is obtained from the composition of $K_{t}$ with $N_{\varphi}$ would be empty, failing to show that $M \models_{\preceq}$.

Consider now the DBW $D_{\varphi}$ for $\varphi$ depicted in Figure 1. The ABW over 1-letter alphabet that is the result of the composition of $M$ and $D_{\varphi}$ makes no guesses regarding the future. Thus, an accepting run of this ABW has two paths. The first path is $\left(s_{0}, d_{0}\right),\left(s_{1}, d_{0}\right),\left(s_{4}, d_{0}\right),\left(s_{5}, d_{0}\right), \ldots$. The second path is $\left(s_{0}, d_{0}\right),\left(s_{1}, d_{0}\right),\left(s_{2}, d_{0}\right),\left(s_{3}, d_{1}\right), \ldots$, where the transition from $\left(s_{2}, d_{0}\right)$ to $\left(s_{3}, d_{1}\right)$ guesses locally that the label of $p_{y}$ in $s_{2}$ is true. Both paths visit accepting states $\left(d_{1}\right.$ and $d_{0}$, respectively) infinitely often. The two paths share the prefix $\left(s_{0}, d_{0}\right),\left(s_{1}, d_{0}\right)$ giving rise to a single run tree of the ABW.

We now proceed to the lower bound. We start with a definition of LTL realizability. Consider a set of propositions $A P=I \cup O$ of input and output signals, respectively. Let $L$ be a language of infinite words over alphabet $2^{A P}$. The realizability problem for $L$ is to decide whether there exists a strategy $f:\left(2^{I}\right)^{+} \rightarrow 2^{O}$ such that all the computations generated by $f$ are in $L$. A computation $\pi=\left(i_{0}, o_{0}\right),\left(i_{1}, o_{1}\right), \ldots$ is generated by $f$ if for all $j \geq 0$ we have $o_{j}=f\left(i_{0} i_{1} \cdots i_{j}\right)$. The realizability problem for an LTL formula $\varphi$ is the realizability problem for $L(\varphi)$.

Theorem 8. [PR89] The realizability problem for an LTL formula $\varphi$ is 2EXPTIME-hard in the size of $\varphi$.

Theorem 9. LTL Generalized model checking $M \models_{\preceq} \varphi$ is 2EXPTIME-hard in the size of $\varphi$.

Proof. We show how to solve realizability of an LTL formula using the generalized model checking problem. The idea behind the reduction is that the PKS includes determined values of the inputs and undetermined values of the outputs. The branching of the PKS forces all possible assignments to inputs as possible successors of every state. Thus, every completion of the PKS associates an assignment to the outputs with every possible assignment to inputs and is in essence a strategy. If the completion satisfies the LTL formula, then so does the strategy. The PKS has $2^{I}$ different states, each labeled by the appropriate assignment to the input variables and with transitions between every two possible states. Formally, we have the following. 

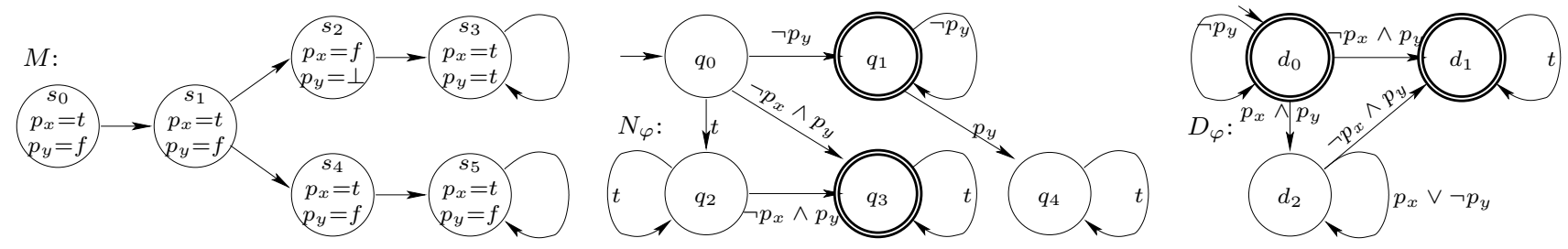

Fig. 1. Partial Kripke Structure $M$ showing the difference between usage of NBW $N_{\varphi}$ and DBW $D_{\varphi}$, where $\varphi=F\left(\neg p_{x} \wedge p_{y}\right) \vee G\left(\neg p_{y}\right)$. Transitions of both automata are labeled with formulas over propositions. In particular, transitions labeled with $t$ read all possible input letters.

Consider the PKS $M=\left\langle S, R, L, s^{i n}\right\rangle$ with $S=2^{I}, R=$ $S \times S$, and $s^{i n} \in S$ is chosen arbitrarily. For every proposition $i \in I$ we have $L(s, i)=$ true if $i \in s$ and $L(s, i)=$ false otherwise. For every proposition $o \in O$ we have $L(s, o)=\perp$. Consider the problem of generalized model checking of $X \varphi$ on $M$.

Suppose that $M \models_{\prec} X \varphi$. There exists $\overline{M^{\prime}}=\left\langle S^{\prime}, R^{\prime}\right.$, $\left.L^{\prime}, s_{0}^{\prime}\right\rangle$ such that $M \preceq \overline{M^{\prime}}$ and $\overline{M^{\prime}} \models X \varphi$. For every state $t \in S^{\prime}$ and every assignment $\sigma: I \rightarrow\{$ true, false $\}$ there exists a state $t^{\prime} \in S^{\prime}$ such that $\left(t, t^{\prime}\right) \in R^{\prime}$ and $L\left(t^{\prime}\right)$ agrees with $\sigma$ on all the propositions in $I$. For $\sigma \in 2^{I}$ let $\sigma(t)$ denote this state $t^{\prime}$. It follows that every sequence $\sigma_{1} \cdots \sigma_{k} \in\left(2^{I}\right)^{+}$ induces a sequence $s_{1}^{\prime}, \ldots, s_{k}^{\prime}$ such that $s_{1}^{\prime}=\sigma_{1}\left(s_{0}^{\prime}\right)$ and $s_{i+1}^{\prime}=\sigma_{i+1}\left(s_{i}^{\prime}\right)$. Denote $s_{1}^{\prime}, \ldots, s_{k}^{\prime}$ by $\sigma_{1} \cdots \sigma_{k}\left(s_{0}^{\prime}\right)$ and let $O\left(s_{1}^{\prime}, \ldots, s_{k}^{\prime}\right)$ denote the projection of $L^{\prime}\left(s_{k}^{\prime}\right)$ on the propositions in $O$. Consider the strategy $f:\left(2^{I}\right)^{+} \rightarrow 2^{O}$ where for $w \in\left(2^{I}\right)^{+}$we set $f(w)=O\left(w\left(s_{0}^{\prime}\right)\right)$. Consider a computation $\pi=\left(i_{1}, o_{1}\right), \ldots$ generated by $f$. By construction $\left(i_{0}, o_{0}\right),\left(i_{1}, o_{1}\right), \ldots$ is a computation of $M$ where $\left(i_{0}, o_{0}\right)=$ $L^{\prime}\left(s_{0}^{\prime}\right)$. As $\overline{M^{\prime}} \models X \varphi$ we conclude that $\pi \models \varphi$.

Suppose that $\varphi$ is realizable. There exists a strategy $f$ : $\left(2^{I}\right)^{+} \rightarrow 2^{O}$ such that every computation induced by $f$ satisfies $\varphi$. Consider the tree $\left\langle\left(2^{I}\right)^{*}, L^{\prime}\right\rangle$ where $L^{\prime}(w \cdot i)=$ $i \cup f(w)$ and $L^{\prime}(\epsilon)$ agrees with $L\left(s^{i n}\right)$ on the propositions in $I$. It is simple to see that $\left\langle\left(2^{I}\right)^{*}, L^{\prime}\right\rangle$ completes $M$ and satisfies $X \varphi$.

This construction results in an exponential PKS. It follows, that a generalized model checking algorithm that is exponential in the input PKS would not constitute a violation to the 2EXPTIME-hardness of LTL realizability. We show how to replace the exponential PKS with a PKS that has two states. The structure uses the propositions $\{p, z\} \cup O$. Let $n=|I|$. A state $s$ in the structure above is represented by a sequence $s_{1} \ldots s_{n}$ of $n$ states. The assignment to $i_{j}$ is the assignment to $p$ in state $s_{j}$ and the assignment to $O$ is the assignment to $O$ in state $s_{n}$. Specifically, $M=\left\langle S, R, L, s_{p}\right\rangle$ where $S=$ $\left\{s_{p}, s_{\bar{p}}\right\}, R=S \times S$, and $L\left(s_{p}, p\right)=$ true,$L\left(s_{\bar{p}}, p\right)=$ false, and $L(s, q)=\perp$ for $s \in S$ and $q \in O \cup\{z\}$. The proposition $z$ is going to mark the beginning of a sequence of $n$ states.

We now rewrite the LTL formula $\varphi$ into a new LTL formula that relativizes the occurrence of an input $i_{j}$ in $\varphi$ to the new location representing the value of $i_{j}$ (by replacing $i_{j}$ by $X^{j-1} p$ ), relativizes the occurrence of an output $o$ in $\varphi$ to the new location $X^{n-1} o$, and relativizes the truth values of all other operators to the locations where $z$ is true. Formally, we define $g: L T L \rightarrow L T L$ as follows.
1. $g\left(i_{j}\right)=X^{j-1} p$ for $i_{j} \in I$

2. $g(o)=X^{n-1} o$ for $o \in O$

3. $g(\varphi \wedge \psi)=g(\varphi) \wedge g(\psi)$

4. $g(\neg \varphi)=\neg g(\varphi)$

5. $g(X \varphi)=X^{n} g(\varphi)$

6. $g(\varphi U \psi)=((z \rightarrow g(\varphi)) U(z \wedge g(\psi)))$

Finally, let

$$
\begin{aligned}
\varphi_{z}=(\neg z \wedge X z) \wedge \\
G(z \rightarrow(\underbrace{X(\neg z \wedge X(\neg z \wedge \ldots \wedge X(\neg z \wedge X}_{n \text { next operators }} z)))))
\end{aligned}
$$

We set $\varphi^{\prime}=\varphi_{z} \wedge X g(\varphi)$. We note that the length of $g(\varphi)$ is linear in $|I| \cdot|\varphi|$ and the length of $\varphi_{z}$ is linear in $|I|$.

We define a transformation on words that takes a word over $\mathbf{2}^{\{p, z\} \cup O}$ and produces a word over $\mathbf{2}^{I \cup O}$ that matches the transformation $g$ for LTL formulas. Consider a word $w=$ $\sigma_{0} \sigma_{1} \cdots \in \mathbf{2}^{\{p, z\} \cup O}$. Let $\bar{g}(w)$ denote the word $w^{\prime}=\sigma_{1}^{\prime} \cdots \in$ $\mathbf{2}^{I \cup O}$ such that $\sigma_{m}^{\prime}\left(i_{j}\right)=\sigma_{(m-1) n+j}(p)$ and $\sigma_{m}^{\prime}(o)=\sigma_{m n}(o)$. Suppose that $w=\varphi_{z}$. For every LTL formula $\varphi$ and every location $n$ we can show by induction on the structure of $\varphi$ that $\bar{g}(w), n \models \varphi$ iff $w,(m-1) n+1 \models g(\varphi){ }^{3}$

Suppose that $\varphi$ is realizable by strategy $f$. We translate $f$ to a labeling of the $\{0,1\}^{*}$ tree in the obvious way. Every node $x 0$ is labeled by $\neg p$ and every node $x 1$ is labeled by $p$. Every node $x$ such that $|x|$ is a multiple of $n$ plus 1 is labeled by $z$ and all others are labeled by $\neg z$. Every node $x$ such that $|x|$ is a multiple of $n$ is labeled by the appropriate assignment to the propositions in $O$. For every other node $x$, the assignment to propositions in $O$ is arbitrary. Consider a path in the resulting tree. It is simple to see that the path satisfies $\varphi_{z}$. As the original path satisfies $\varphi$ we can show that the result satisfies $X g(\varphi)$ as well.

Suppose that there is a structure $K$ that completes $M$ such that $K \models \varphi^{\prime}$. Consider the unwinding $K^{+}$of $K$. As $K$ is complete, so is $K^{+}$. Let $K^{+}=\left\langle T, R^{\prime}, L^{\prime}, s^{i n}\right\rangle$. We abuse notation and denote by $K^{+}$a pruning of $K^{+}$that includes for every state $t \in T$ exactly one state $t^{\prime}$ such that $\left(t, t^{\prime}\right) \in R^{\prime}$ and $p \in L^{\prime}\left(t^{\prime}\right)$ and exactly one state $t^{\prime \prime}$ such that $\left(t, t^{\prime \prime}\right) \in R^{\prime}$ and $p \notin L^{\prime}\left(t^{\prime \prime}\right)$. In addition, we identify a sequence $\pi \in$ $\{0,1\}^{*}$ with the state $t \in T$ such that $\epsilon$ is identified with $s^{i n}$ and $\pi 1$ is identified with the successor $t^{\prime}$ of $\pi$ such that $p \in$ $L^{\prime}\left(t^{\prime}\right)$ and $\pi 0$ is identified with the successor $t^{\prime \prime}$ of $\pi$ such that $p \notin L^{\prime}\left(t^{\prime \prime}\right)$. Consider a letter $\sigma \in 2^{I}$, we identify $\sigma$ with the sequence in $\alpha_{1} \cdots \alpha_{n} \in\{0,1\}^{n}$ such that $\alpha_{j}=1$ iff $i_{j} \in \sigma$.

\footnotetext{
${ }^{3}$ Note that $w$ starts from location 0 and $t(w)$ from location 1.
} 
Finally, we use the same notation for sequences in $\left(2^{I}\right)^{*}$. We are now ready to define the strategy $f:\left(2^{I}\right)^{*} \rightarrow 2^{O}$. For a sequence $\pi \in\left(2^{I}\right)^{*}$ we set $f(\pi)=L_{O}^{\prime}(\pi)$. It is simple to see that every computation induced by $f$ satisfies $\varphi$.

We note that it is possible also to reduce the number of propositions used in $M$ to three by doing a similar encoding for the outputs using a single proposition $q$. The cycle induced by $z$ has to grow to size $|I \cup O|$ and in addition the formula has to be modified to notice just the path labeled by $p$ in the positions $(|I|+1), \ldots,(|I|+|O|)$.

By Theorems 7 and 9 we have the following.

Corollary 2. LTL Generalized model checking $M \models \preceq \varphi$ is 2EXPTIME-complete in the size of $\varphi$.

\section{Linear Completeness Preorder}

The completeness preorder $\preceq$ used to define the generalized model checking relation $=\prec$ is stronger than necessary for reasoning only about the linear behaviors of partial Kripke structures. Indeed, the completeness preorder reduces to a bisimulation relation in the case of complete Kripke structures, and Kripke structures that satisfy the same LTL formulas are not necessarily bisimilar.

In this section, we study a simpler linear completeness preorder $\preceq_{L}$, first suggested in [BG00], that relates partial Kripke structures using only their sets of (3-valued) traces. Then we show that generalized model checking $\models_{{ }_{L}}$ defined with respect to this linear preorder is "only" EXPSPACEcomplete.

Given two infinite 3-valued traces $w=L\left(s_{0}\right) L\left(s_{1}\right) \cdots$ and $w^{\prime}=L\left(s_{0}^{\prime}\right) L\left(s_{1}^{\prime}\right) \cdots$ in $\left(\mathbf{3}^{A P}\right)^{\omega}$, we write $w \leq_{I} w^{\prime}$ if $\forall i \geq 0$ : $\forall p \in A P: L\left(s_{i}, p\right) \leq_{I} L\left(s_{i}^{\prime}, p\right)$.

Definition 2. For two PKS $M_{i}=\left\langle S_{i}, R_{i}, L_{i}, s_{i}^{i n}\right\rangle$ with $i=$ 1,2 , the linear completeness preorder $\preceq_{L}$ is the greatest relation $\preceq_{L} \subseteq S_{1} \times S_{2}$ such that $\left(s_{1}, s_{2}\right) \in \preceq_{L}$ implies all the following.

1. For every $w \in \mathcal{L}\left(M_{1}, s_{1}\right)$ there exists $w^{\prime} \in \mathcal{L}\left(M_{2}, s_{2}\right)$ such that $w \leq_{I} w^{\prime}$.

2. For every $w^{\prime} \in \mathcal{L}\left(M_{2}, s_{2}\right)$ there exists $w \in \mathcal{L}\left(M_{1}, s_{1}\right)$ such that $w \leq_{I} w^{\prime}$.

It is easy to show that 3-valued LTL logically characterizes the linear completeness preorder.

Theorem 10. For every two PKS $M_{1}$ and $M_{2}$, we have $M_{1} \preceq_{L}$ $M_{2}$ iff for every LTL formula $\varphi$ we have $\left[M_{1} \models \varphi\right] \leq_{I}$ $\left[M_{2} \models \varphi\right]$.

Proof. Assume $M_{1} \preceq_{L} M_{2}$ and consider an LTL formula $\varphi$. If $\left[M_{1} \models \varphi\right]=\perp$, we always have $\left[M_{1} \models \varphi\right] \leq_{I}\left[M_{2} \models \varphi\right]$.

If $\left[M_{1} \models \varphi\right]=$ true, then for all $w \in \mathcal{L}\left(M_{1}\right),[w \models$ $\varphi]=$ true. By point 2 of Definition 2 , for every $w^{\prime} \in \mathcal{L}\left(M_{2}\right)$ there exists $w \in \mathcal{L}\left(M_{1}\right)$ such that $w \leq_{I} w^{\prime}$. But since $\forall w \in$
$\mathcal{L}\left(M_{1}\right):[w \mid=\varphi]=$ true, we have $\forall w^{\prime} \in \mathcal{L}\left(M_{2}\right):\left[w^{\prime} \models\right.$ $\varphi]=$ true, and hence $\left[M_{2} \mid=\varphi\right]=$ true.

If $\left[M_{1} \models \varphi\right]=$ false, then $\exists w \in \mathcal{L}\left(M_{1}\right):[w \models \varphi]=$ false. By point 1 of Definition 2, we have $\exists w^{\prime} \in \mathcal{L}\left(M_{2}\right)$ : $w \leq_{I} w^{\prime}$ and hence $\left[w^{\prime} \models \varphi\right]=$ false. Thus $\left[M_{2} \models \varphi\right]=$ false, and the first direction of the theorem holds.

Conversely, let $s_{1} \sqsubseteq s_{2}$ denote $\forall \varphi \in L T L:\left[\left(M_{1}, s_{1}\right) \models\right.$ $\varphi] \leq_{I}\left[\left(M_{2}, s_{2}\right) \models \varphi\right]$. Assume that $s_{1} \sqsubseteq s_{2}$ but that $s_{1} \npreceq_{L}$ $s_{2}$ : thus, either point 1 or 2 of Definition 2 is violated.

Assume point 1 is violated: $\exists w \in \mathcal{L}\left(M_{1}, s_{1}\right): \forall w^{\prime} \in$ $\mathcal{L}\left(M_{2}, s_{2}\right): w \underline{Z}_{I} w^{\prime}$. Let $w=s_{1}^{0} s_{1}^{1} s_{1}^{2} \cdots$ with $s_{1}^{0}=s_{1}$. Let $S_{2}^{0}=\left\{s_{2}\right\}$ and for $k>0$, let $S_{2}^{k}=\left\{s \in S_{2} \mid s^{\prime} \in S_{2}^{k-1} \wedge\right.$ $\left.\left(s^{\prime}, s\right) \in R_{2} \wedge\left(\forall p \in A P: L_{1}\left(s_{1}^{k}, p\right) \leq_{I} L_{2}(s, p)\right)\right\}$. Since $\forall w^{\prime} \in \mathcal{L}\left(M_{2}, s_{2}\right): w \mathbb{L}_{I} w^{\prime}$, then there must exist a value of $k$ such that $S_{2}^{k}=\emptyset$. In other words, the corresponding $s_{1}^{k}$ in $M_{1}$ denote the first state in $M_{1}$ reachable from $s_{1}$ along $w$ whose label cannot be "matched" (according to the previous formal definition) by even one state of $M_{2}$ (hence also reachable in $k$ steps from $s_{2}$ ). We identified $k$ such that $S_{s}^{k}=\emptyset$. Let $T_{2}^{k}=\left\{s \in S_{2} \mid s^{\prime} \in S_{2}^{k-1} \wedge\left(s^{\prime}, s\right) \in R_{2}\right\}$ (by construction, we know $S_{2}^{k-1} \neq \emptyset$ and since every state has at least one successor state, $T_{2}^{k}$ is nonempty as well). Thus, for each state $s \in T_{2}^{k}$, there exists a proposition $p \in A P$ such that $L_{1}\left(s_{1}^{k}, p\right) \not \leq_{I} L_{2}(s, p)$. Let $\varphi(s)=p$ if $L_{1}\left(s_{1}^{k}, p\right)=$ false and let $\varphi(s)=\neg p$ otherwise (i.e., when $L_{1}\left(s_{1}^{k}, p\right)=$ true; if $L_{1}\left(s_{1}^{k}, p\right)=\perp$, then trivially $\left.L_{1}\left(s_{1}^{k}, p\right) \leq_{I} L_{2}(s, p)\right)$. Consider the LTL formula

$$
\begin{aligned}
& \psi=\left(\bigwedge_{i<k}\left(X^{i}\left(\varphi_{L\left(s_{1}^{i}\right)}\right)\right)\right) \Rightarrow X^{k} \bigvee_{s \in T_{2}^{k}} \varphi(s), \\
& \text { where } \varphi_{L\left(s_{1}^{i}\right)}=\bigwedge_{L\left(s_{1}^{i}, p\right)=\text { true }} p \wedge \bigwedge_{L\left(s_{1}^{i}, p\right)=\text { false }} \neg p
\end{aligned}
$$

We have $\left[\left(M_{1}, s_{1}\right) \models \psi\right]=$ false (as we know $[w \models \psi]=$ false) while $\left[\left(M_{2}, s_{2}\right)=\psi\right] \neq$ false (since the antecedent of the logical implication is true exactly for finite paths leading to states in $S_{2}^{k-1}$ and the consequent is either true or $\perp$ for all states in $T_{2}^{k}$ ). A contradiction with $s_{1} \sqsubseteq s_{2}$.

Assume point 2 is violated: $\exists w^{\prime} \in \mathcal{L}\left(M_{2}, s_{2}\right): \forall w \in$ $\mathcal{L}\left(M_{1}, s_{1}\right): w \not_{I} w^{\prime}$. Using the same line of reasoning as in the previous case, let $s_{2}^{k}$ denote the first state in $M_{2}$ reachable from $s_{2}$ along $w^{\prime}$ whose label cannot be matched by even one state in $S_{1}^{k}$ of $M_{1}$ as defined above. Thus, for each state $s \in$ $S_{1}^{k}$, there exists a proposition $p \in A P$ such that $L_{1}(s, p) \mathbb{Z}_{I}$ $L_{2}\left(s_{2}^{k}, p\right)$. Let $\varphi(s)=p$ if $L_{1}(s, p)=$ true and let $\varphi(s)=$ $\neg p$ otherwise. Consider the LTL formula

$$
\psi=\left(\bigwedge_{i<k}\left(X^{i}\left(\varphi_{L\left(s_{2}^{i}\right)}\right)\right)\right) \Rightarrow X^{k} \bigvee_{s \in S_{1}^{k}} \varphi(s),
$$

We have $\left[\left(M_{1}, s_{1}\right)=\psi\right]=$ true (since the antecedent of the logical implication is either true or $\perp$ exactly for the finite paths leading to states in $S_{1}^{k-1}$ and the consequent is true for all states in $\left.S_{1}^{k}\right)$ while $\left[\left(M_{2}, s_{2}\right) \models \psi\right] \neq$ true (since $\left[w^{\prime} \models \psi\right] \neq$ true $)$. A contradiction with $s_{1} \sqsubseteq s_{2}$. 
Given a PKS $M$ and an LTL formula $\varphi$, generalized model checking with respect to the linear completeness preorder $\preceq_{L}$ means checking whether every 3 -valued trace of $M$ can be completed to a 2-valued trace that satisfies $\varphi$. Formally, we have the following.

$$
\begin{aligned}
& M \models \preceq_{L} \varphi \text { iff } \forall w \in \mathcal{L}(M): \\
& \quad \exists \text { a complete } w^{\prime} \text { such that } w \leq_{I} w^{\prime} \text { and } w^{\prime} \models \varphi
\end{aligned}
$$

As observed in [GJ02], computing the value of $[M \models$ $\varphi]_{t}$ for an LTL formula $\varphi$ can be reduced to one normal (2valued) model checking problem and one generalized model checking problem, regardless of which completeness preorder is used. One can start by checking whether there exists a completion $w^{\prime}$ of every trace $w$ in $M$ such that $w^{\prime} \not \forall \varphi$. To do this, one can build a Kripke structure $M^{c}$ that guesses all possible completions of labelings of states of $M$ and thus accepts all the possible completions of traces of $M$. Then, one checks whether $M^{c} \models \varphi$ using traditional 2-valued LTL model checking, which is a PSPACE-complete problem. If $M^{c} \models \varphi$, all possible completions of $M$ satisfy $\varphi$, which means $[M \models \varphi]_{t}=$ true and we stop. Otherwise, one needs to solve a second, more expensive generalized model checking problem to determine whether there exists some completion $M^{\prime}$ of $M$ whose traces all satisfy $\varphi$.

If one considers the completeness preorder $\preceq$, checking for such a completion $M^{\prime} \succeq M$ such that $M^{\prime} \models \varphi$, i.e., computing $M \models_{\preceq} \varphi$, is 2EXPTIME-complete as shown in the previous section. However, if one considers instead the linear completeness preorder $\preceq_{L}$, we now show that computing $M \models_{\preceq_{L}} \varphi$ is only EXPSPACE-complete.

Theorem 11. LTL generalized model checking $M \models_{\Omega_{L}} \varphi$ with respect to the linear completeness preorder $\preceq_{L}$ can be solved in space logarithmic in the size of $M$ and exponential in the size of $\varphi$.

Proof. Consider an LTL formula $\varphi$. According to Theorem 3 there exists an NBW $N_{\varphi}=\left\langle\mathbf{2}^{A P}, Q, q_{0}, \rho, F\right\rangle$ where $|Q|=$ $2^{O(|\varphi|)}$ such that $L\left(N_{\varphi}\right)=L(\varphi)$.

We modify the NBW above to an NBW over the alphabet $3^{A P}$ that accepts partial traces that have a completion in $L\left(N_{\varphi}\right)$. Formally, we have the following.

We denote letters in $2^{A P}$ by $\bar{\sigma}$ and letters in $3^{A P}$ by $\tau$. Let $N^{\prime}$ be the automaton obtained from $N_{\varphi}$ by guessing a completion of the read letter. Formally, $N^{\prime}=\left\langle\mathbf{3}^{A P}, Q, q_{0}, \rho^{\prime}, F\right\rangle$ where

$$
\rho^{\prime}(s, \tau)=\bigvee_{\bar{\sigma} \succeq \tau} \rho(s, \bar{\sigma})
$$

Now, all that we have to check is whether $L(M) \subseteq L\left(N^{\prime}\right)$. From Theorem 6, we know that this problem can be solved in space logarithmic in $M$ and polynomial in $N^{\prime}$. As $N^{\prime}$ is exponential in $\varphi$, the upper bound follows.

Example 3. We revisit the partial Kripke structure $M$ depicted in Figure 1. Let $\varphi=F\left(\neg p_{x} \wedge p_{y}\right) \vee G\left(\neg p_{y}\right)$. We recall that $M \models_{\preceq} \varphi$ and validate that $M \models_{\preceq_{L}} \varphi$. This time, it is sufficient to use the automaton $N_{\varphi}$ in Figure 1.
The construction calls for replacing a transition over $\bar{\sigma}$ with transitions over $\sigma$ for every $\sigma \preceq \bar{\sigma}$. In this case, a transition from $q_{2}$ to $q_{3}$ is added reading the letter $\neg p_{x} \wedge\left(p_{y}=\perp\right)$. With this transition in place, the run $q_{0}, q_{2}, q_{2}, q_{3}, \ldots$ accepts the trace generated by $s_{0}, s_{1}, s_{2}, s_{3}, \ldots$ Using the existing transitions, the run $q_{0}, q_{1}, q_{1}, q_{1}, \ldots$ accepts the trace generated by $s_{0}, s_{1}, s_{4}, s_{5}, \ldots$.

We now show that using this definition of GMC we can solve an EXPSPACE-hard tiling problem [vEB97]. In tiling problems we get a finite set of different types of tiles and we have to tile a floor of a given dimension. We may use as many tiles as we want from every given type, however, there are rules that tell us which tiles are allowed to be next to each other according to vertical and horizontal rules. There are many different flavors of tiling problems with different complexities. Here we introduce the EXPSPACE version of the tiling problem. In order to prove the lower bound, we build a PKS $M$ whose traces are all the possible arrangements of tiles. A trace has a completion that satisfies our LTL formula $\varphi$ if the arrangement of tiles is not valid, i.e., it violates one of the tiling rules. That is, $M=_{\preceq_{L}} \varphi$ iff all possible arrangements of tiles are not valid, i.e., the tiling problem does not have a solution.

A tiling problem is $\langle T, H, V, s, t, n\rangle$, where $T$ is a finite set of tiles, $H, V \subseteq T \times T$ are horizontal and vertical consistency rules, $s, t \in T$ are initial and final tiles, and $n$ is a number (in unary). The decision problem is whether there exists a number $m$ and a function $f:\left[2^{n}\right] \times[m] \rightarrow T$ such that $f(1,1)=s, f\left(2^{n}, m\right)=t$, and forall $i, j$ we have $(f(i, j), f(i+1, j)) \in H$ and $(f(i, j), f(i, j+1)) \in$ $V$. That is, arrange the tiles in a $2^{n}$ times $m$ rectangle such that $s$ is in the bottom left corner, $t$ in the top right corner, and all neighbors (vertical/horizontal) satisfy the horizontal and vertical consistency rules. This problem is EXPSPACEcomplete [vEB97].

Theorem 12. LTL generalized model checking $M \models_{\preceq_{L}}$ with respect to the linear completeness preorder $\preceq_{L}$ is EXPSPACEhard in the size of $\varphi$.

Proof. We start by representing the rectangular arrangement of tiles by a linear sequence of tiles. An (infinite) linear sequence of tiles represents a valid tiling if it starts with $s$, has $t$ in location $m 2^{n}$ for some $m$, every adjacent locations (except multiples of $2^{n}$ and their successors) satisfy $H$, and every two locations whose distance is $2^{n}$ satisfy $V$.

We construct a simple system that produces all possible sequences of tiles. The partial propositions are going to number every tile in the sequence with a number in $\left[0 . .\left(2^{n}-\right.\right.$ 1)]. The LTL formula checks two things. First, that the truth assignments to partial propositional variables behave like a counter (it is always possible to complete the values of these propositions in this way). Second, that every possible sequence of tiles contains one of the following problems: either (a) it does not start in $s$, or (b) all locations that are multiples of $2^{n}$ are not $t$, or (c) the horizontal rule is violated before $t$ appears in a $2^{n}$-multiple location, or (d) the vertical rule is 
violated before $t$ appears in a $2^{n}$-multiple location. If one of these problems occurs, then the tiling is not valid. If all possible arrangements of tiles are not valid, then the tiling problem does not have a solution.

Suppose that $|T|=I=2^{k}$, then the system is the $I$ clique. We have $k$ propositions $\left\{p_{1}, \ldots, p_{k}\right\}$ and every state encodes one possible tile. The initial state is the state that corresponds to $s$. We have $n$ propositions $\left\{q_{1}, \ldots, q_{n}\right\}$ that encode the location of the tile modulo $2^{n}$. Finally, we have two more propositions $\{a, b\}$ that are used to identify the location of a violation of the vertical rule. In all states of the system the value of $\left\{a, b, q_{1}, \ldots, q_{n}\right\}$ is unknown. Formally, let $M=\langle S, R, L, s\rangle$ where $S=2^{\left\{p_{1}, \ldots, p_{k}\right\}}, R=S \times S$, $L\left(t, p_{i}\right)=$ true if $p_{i} \in t, L\left(t, p_{i}\right)=$ false if $p_{i} \notin t, L(t, q)=$ $\perp$ for $q \in\left\{a, b, q_{1}, \ldots, q_{n}\right\}$, and by abuse of notation $s$ is the state that corresponds to the initial tile $s$.

The LTL formula is $\varphi=\varphi_{1} \wedge \varphi_{2}$, where $\varphi_{1}$ and $\varphi_{2}$ are defined below.

1. Counter consistency $-\varphi_{1}$ is conjunction of the following formulas.

(a) The counter starts at 0 :

$$
\bigwedge_{j=1}^{n} \neg q_{j}
$$

(b) The counter respects normal counting:

$$
\begin{aligned}
& G\left(q_{1} \leftrightarrow X \neg q_{1}\right) \\
& G\left(\bigwedge_{j=2}^{n}\left(\left(\bigwedge_{j^{\prime}<j} q_{j^{\prime}}\right) \rightarrow\left(q_{j} \leftrightarrow X \neg q_{j}\right)\right)\right)^{\wedge} \\
& G\left(\bigwedge_{j=2}^{n}\left(\left(\bigvee_{j^{\prime}<j} \neg q_{j^{\prime}}\right) \rightarrow\left(q_{j} \leftrightarrow X q_{j}\right)\right)\right)
\end{aligned}
$$

2. The formula $\varphi_{2}$ describes the possible problems in the sequence of tiles. Let $\phi_{e}$ stand for $\left(\bigwedge_{j=1}^{n} q_{j}\right)$, i.e., the counter is $2^{n}-1$ and let $\phi_{t}$ stand for $\left(\phi_{e} \rightarrow \neg t\right)$, i.e., if the counter is $2^{n}-1$ the tile is not $t$. The formula $\varphi_{2}$ is the disjunction of the following formulas.

(a) The sequence does not start with tile $s$ : $\neg s$.

(b) Every $2^{n}$ multiple is not marked with tile $t$ : $G \phi_{t}$.

(c) The horizontal rule is violated somewhere that is not the end of a configuration and before the appearance of $t$ at the end of a configuration:

$$
\left(\neg \phi_{e} \wedge\left(\bigwedge_{\left(p, p^{\prime}\right) \in H} \neg p \vee X \neg p^{\prime}\right)\right) R \phi_{t}
$$

(d) The propositions $a$ and $b$ are used to find a violation of the vertical rules. The disjunct of $\varphi_{2}$ that expresses a violation of the vertical rule is the conjunction of the following:

i. The proposition $a$ is assigned true exactly once, and $b$ is assigned true after it: $(\neg a) U(a \wedge X G \neg a \wedge X F b)$. ii. The proposition $b$ is assigned true exactly once: $(\neg b) U(b \wedge X G \neg b)$.

iii. The location where $b$ is assigned true occurs before a location where $t$ marks a location that is a multiple of $2^{n}-1: b R \phi_{t}$.

iv. The locations where $a$ and $b$ are true agree on the counter valuation: $G\left(a \rightarrow \bigwedge_{j=1}^{n}\left(q_{j} \leftrightarrow F\left(b \wedge q_{j}\right)\right)\right)$.

$\mathrm{v}$. There is exactly one occurrence of $2^{n}-1$ between the locations where $a$ and $b$ are assigned true: $G(a \rightarrow$ $\left.\neg \phi_{e} U\left(\phi_{e} \wedge X\left(\neg \phi_{e} U b\right)\right)\right)$

vi. The locations marked by $a$ and $b$ violate the vertical consistency:

$$
\bigwedge_{\left(p, p^{\prime}\right) \in V}\left(F(a \wedge p) \rightarrow G\left(b \rightarrow \neg p^{\prime}\right)\right)
$$

Lemma 2. We have $M \models_{\preceq_{L}} \varphi$ iff $T$ does not have a solution.

Proof. Suppose that $M \models_{\preceq_{L}} \varphi$. It follows that for every possible sequence of tiles, we can find a truth assignment to the propositions in $\left\{a, b, q_{1}, \ldots, q_{n}\right\}$ such that this extension satisfies $\varphi$. It follows that the behavior of the propositions $\left\{q_{1}, \ldots, q_{n}\right\}$ is completely deterministic and simulates a $2^{n}$ counter. Now, one of the disjuncts of $\varphi_{2}$ does not hold. It follows that there is some problem with the arrangement of tiles. Thus, all possible sequences of tiles are not valid and the tiling problem $T$ does not have a solution.

In the other direction, suppose that the tiling problem $T$ does not have a solution. Then, for every possible sequence or tiles there exists a problem with one of the tiling rules. We add the assignment of the propositions $\left\{a, b, q_{1}, \ldots, q_{n}\right\}$ according to this problem.

In this construction the number of states of $M$ is linear in the number of tiles of $T$ and it uses $n$ propositions. We show how to replace $M$ with a system $M^{\prime}$ of a constant size that uses a constant number of propositions. The modifications to the LTL formula $\varphi$ resemble those outlined above in the proof of Theorem 7. We are going to use the following propositions. 1. $z$ - partial info - marks the location of the beginning of every tile.

2. $p$ - full info - used to encode the name of the tile.

3. $q$ - partial info - used to encodes the number of a tile module $2^{n}$.

4. $a, b$ - partial info - mark the locations where a vertical rule is violated (as above).

The system $M^{\prime}$ is the 2-state clique. One state is labeled $p$ and the other $\neg p$. The state labeled $p$ is initial. Formally, $M^{\prime}=$ $\left\langle S^{\prime}, R^{\prime}, L^{\prime}, s_{p}\right\rangle$, where $S^{\prime}=\left\{s_{p}, s_{\bar{p}}\right\}, R^{\prime}=S^{\prime} \times S^{\prime}$, and $L\left(s_{p}, p\right)=$ true, $L\left(s_{\bar{p}}, p\right)=$ false, and $L(s, r)=\perp$ for $s \in$ $S$ and $r \in\{q, a, b, z\}$. Wlog, we assume that $n>i$. Then, a sequence of $n$ states of this system encodes one tile and its number. Thus, a sequence of $n$ states of $M^{\prime}$ corresponds to one state of $M$. The value of the proposition $p$ in the states $i+1, \ldots, n$ is not used and thus there are many traces of $M^{\prime}$ 
that correspond to the same tiling. The case where $n \leq i$ is simple to construct using the same ideas.

We change the LTL formula $\varphi$ into a new LTL formula that replaces the name of a tile with reference to a sequence of $k$ truth values to proposition $p$, relativizes the value of the proposition $q_{i}$ to the location representing the value of $q_{i}$ (by replacing $q_{i}$ by $X^{i-1} q$, and relativizes the truth values of all other operators to the locations where $z$ is true. Formally, the function $g: L T L \rightarrow L T L$ is very similar to the function defined in the proof of Theorem 9 and is defined as follows.

1. Let $p_{1}, \ldots, p_{k}$ denote the binary encoding of the tile $t$. Let $P_{i}$ be $X^{i-1} p$ if $p_{i}$ is true and $X^{i-1} \neg p$ if $p_{i}$ is false. Let $g(t)=\bigwedge_{i=1}^{k} P_{i}$ (note that $g(t)$ can be written with $k$ next operators and not $k^{2}$ as above).

2. $g\left(q_{i}\right)$ be $X^{i-1} q$ for $q_{i} \in\left\{q_{1}, \ldots, q_{n}\right\}$

3. $g(r)=r$ for $r \in\{a, b\}$

4. $g(\varphi \wedge \psi)=g(\varphi) \wedge g(\psi)$

5. $g(\neg \varphi)=\neg g(\varphi)$

6. $g(X \varphi)=X^{n} g(\varphi)$

7. $g(\varphi U \psi)=((z \rightarrow g(\varphi)) U(z \wedge g(\psi)))$

Finally, let

$$
\begin{aligned}
& \varphi_{z}=(\neg z \wedge X z) \wedge \\
& G(z \rightarrow(\underbrace{X \neg z \wedge X(\neg z \wedge \ldots \wedge X(\neg z \wedge X}_{n \text { next operators }} z))))
\end{aligned}
$$

We set $\varphi^{\prime}=\varphi_{z} \wedge X g(\varphi)$. We note that the length of $\varphi^{\prime}$ is linear in $n|\varphi|$.

The proof that $M^{\prime} \models_{\swarrow_{L}} \varphi^{\prime}$ iff the tiling problem has no solution combines the proof of Lemma 2 with the techniques in the proof of Theorem 9 .

By Theorems 11 and 12 we have the following.

Corollary 3. LTL Generalized Model Checking $M \mid \models_{\preceq_{L}}$ with respect to the linear completeness preorder $\preceq_{L}$ is EXPSPACEcomplete in the size of $\varphi$.

The next theorem states that $\preceq$ is a stronger relation than $\preceq_{L}$.

Theorem 13. For every partial Kripke structures $M, M^{\prime}$ and LTL formula $\varphi, M \preceq M^{\prime}$ implies $M \preceq_{L} M^{\prime}$, and therefore $M \models \preceq \varphi$ implies $M \models \preceq_{L} \varphi$.

Proof. Immediate from the definitions of $\preceq$ and ${ }_{L}$.

Note that $\preceq$ is strictly stronger than $\preceq_{L}$, as the converse of the theorem does not hold. To illustrate this, consider the LTL formula $\varphi=(p \wedge X p) \vee(\neg p \wedge X \neg p)$ and the partial Kripke structure $M$ in Figure 2. Formally, let $M=\left\langle\left\{s_{0}\right.\right.$, $\left.\left.s_{1}, s_{2}\right\},\left\{\left(s_{0}, s_{1}\right),\left(s_{0}, s_{2}\right),\left(s_{1}, s_{1}\right),\left(s_{2}, s_{2}\right)\right\}, L, s_{0}\right\rangle$ labeled with a single atomic proposition $p$ such that $L\left(s_{0}, p\right)=\perp$, $L\left(s_{1}, p\right)=$ true and $L\left(s_{2}, p\right)=$ false. It is easy to see that $\left[\left(M, s_{0}\right) \models \varphi\right]=\perp$. Moreover, we have $\left(M, s_{0}\right) \quad \models_{\preceq_{L}}$ $\varphi$, as every 3 -valued trace generated from $\left(M, s_{0}\right)$ can be completed by some 2 -valued trace that satisfies $\varphi$. However,

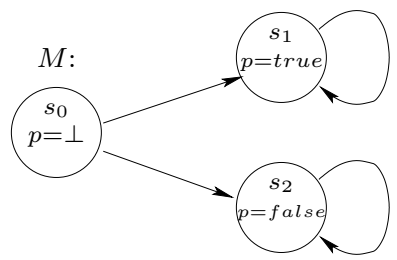

Fig. 2. Partial Kripke Structure showing that $\preceq$ is strictly stronger than $\preceq_{L}$.

$\left(M, s_{0}\right) \not \models \preceq \varphi$ as there does not exist a completion $M^{\prime}$ such that $M \preceq M^{\prime}$ and $M^{\prime} \models \varphi$, as state $s_{0}$ where $p=\perp$ cannot be completed to a single state $s$ such that every trace from $s$ satisfies $\varphi$ : if $L(s, p)=$ true, then the trace $s s_{2}^{\omega}$ violates $\varphi$, and if $L(s, p)=$ false, then the trace $s s_{1}^{\omega}$ violates $\varphi$.

\section{Model Complexity}

We have seen that LTL generalized model checking defined with the stronger branching-time preorder $\preceq$ is polynomial in the size of the model. The degree of the polynomial, however, is unbounded, and depends on the deterministic automaton created for the formula. Here we show that for interesting classes of properties, the model complexity can be restricted to linear or quadratic. The resemblance pointed out between generalized model checking and realizability in the proof of Theorem 9 continues here. Indeed, the same classes of formulas are used to suggest tractable fractions of LTL for realizability (cf. [RW89, AMPS98,PPS06]).

We start with a few additional definitions and known results regarding automata. Let $\mathcal{A}=\left\langle\Sigma, Q, q_{\text {in }}, \delta, \alpha\right\rangle$ be a Büchi automaton. We say that $\mathcal{A}$ is weak if there is a preorder $\leq$ on the state set $Q$ such that the following two conditions hold:

1. For every $q \in Q$ and $\sigma \in \Sigma$, if $q^{\prime}$ appears in $\delta(q, \sigma)$ then $q \leq q^{\prime}$.

2. For every $q \in Q$, if $q \in \alpha$ then forall $q^{\prime}$ such that $q \leq q^{\prime}$ and $q^{\prime} \leq q$ we have $q^{\prime} \in \alpha$.

We use the acronyms mentioned previously for weak automata. For instance, an AWT is an alternating weak tree automaton and an DWW is a deterministic weak word automaton. We specialize Theorem 5 to our needs as follows.

Theorem 14. Given an APW A over a 1-letter alphabet, we can decide whether $L(A)=\emptyset$ in linear time if $A$ is $A W W$ [KVW00] and in quadratic time if $A$ is an $A B W, A C W$, or an $A P W$ with three priorities [VW86, Jur00].

Consider an LTL formula $\varphi$. We say that $\varphi$ is a safety property if for every word $w \notin L(\varphi)$ there exists a prefix $u$ such that forall $v^{\prime}$ we have $u v^{\prime} \notin L(\varphi)$. Let $p$ and $q$ be Boolean combinations of propositional formulas. Formulas of the form $G F p$ or $G(q \rightarrow F p)$ are called response properties, and formulas of the form $F G p$ are called persistence properties [MP92]. If $\varphi$ is of the form $\left(\varphi_{s}^{a} \wedge \varphi_{r}^{a}\right) \rightarrow\left(\varphi_{s}^{g} \wedge \varphi_{r}^{g}\right)$ where $\varphi_{s}^{a}$ and $\varphi_{s}^{g}$ are conjunctions of safety properties and $\varphi_{r}^{a}$ and $\varphi_{r}^{g}$ are conjunctions of response properties is called generalized reactivity [1] [KPP03]. Alternatively, we classify 
LTL properties according to the type of deterministic automaton that accepts the same language. We say that $\varphi$ is a weak property if there exists a DWW that accepts the language of $\varphi$. We say that $\varphi$ is a $D B W$ property if there exists a DBW that accepts the language of $\varphi$. Similarly, we say that $\varphi$ is a $D C W$ property if there exists a DCW that accepts the language of $\varphi$. The following theorem links the different types of LTL properties to the deterministic automata that accept them.

Theorem 15. 1. For every safety property $\varphi$, there exists a $D W W D$ such that $L(D)=L(\varphi)$ [MP90].

2. For every response property $\varphi$, there exists a $D B W D$ such that $L(D)=L(\varphi)$ [MP90].

3. For every persistence property $\varphi$, there exists a DCW $D$ such that $L(D)=L(\varphi)$ [MP90].

4. For every generalized reactivity[1] property $\varphi$, there exists a DPW $D$ with three priorities such that $L(D)=L(\varphi)$ [KPP03].

The following is a consequence of Theorems 14 and 15 and the proof of Theorem 7 .

Corollary 4. LTL generalized model checking $M \models \prec \varphi$ is linear in $M$ for weak and safety properties, and quadratic in $M$ for response, persistence, and generalized reactivity[1] properties.

Proof. From the proof of Theorem 7 it follows that we combine a deterministic automaton for the property with the model to get an APW over a 1-letter alphabet. From Theorem 15 it follows that if the LTL property is a safety or obligation property the DPW, and the resulting APW, are weak. If the LTL property is a response property, the DPW is in fact a DBW. If the LTL property is a persistence property, the DPW is in fact a DCW. If the LTL property is a generalized reactivity[1] property, the DPW has three priorities. Recall that the APW is the product of the DPW and the model. Thus, the APW is linear in the size of the model. The desired upper bound now follows directly from Theorem 14

Note that LTL GMC for persistence properties can be solved in quadratic time in the size of the model, instead of in linear time as incorrectly stated in Theorem 5 of [GJ02]. The root cause of this error is the same as the one for Theorem 25 of [BG00], as the proofs of both theorems rely on the same product construction, now corrected in Theorem 7 of this paper.

Finally, we clarify a subtle misconception regarding generalized model checking of CTL properties. Given a CTL property, we can construct directly an NBT that is at most exponential in the size of the property that accepts all trees that satisfy the property [KVW00]. Generalized model checking can then be solved by combining this NBT with the model to obtain an ABW over a 1-letter alphabet [BG00]. According to Theorem 14 the emptiness of this ABW can be established in quadratic time. Thus, the complexity of GMC with respect to CTL properties is exponential in the formula and quadratic in the model, which is optimal [BG00]. As with
LTL the quadratic complexity in the model follows from the type of acceptance condition used by the automaton for the formula. We are interested in classes of properties for which automata require simpler acceptance conditions. If the CTL property can be recognized by an NWT, the complexity in the size of the model reduces to linear. In the proof of Theorem 7 of [GJ02] it is assumed that if a CTL property can be recognized by an NCT then it can also be recognized by an NWT. However, it is currently unknown whether this is the case (cf. Section 7) and the proof of that theorem is therefore incomplete.

\section{Reducing GMC to Games}

The proof of Theorem 9 reduces realizability of LTL to GMC. The similarity actually goes in both directions. A GMC problem can be translated to a 2-person game where the specification (in LTL or in branching-time logic) can be translated to the winning condition. In a 2-person game players verifier and refuter alternate in moving a token along the edges of a graph. If the infinite path made by the token satisfies an LTL formula, verifier wins and otherwise she loses. If the winning condition is expressed in terms of branching-time logic, instead of considering a path in the graph, we consider the infinite unwinding of the game graph and prune the unwinding so that nodes that correspond to decisions of verifier have exactly one successor. Intuitively, the translation of the GMC problem to such a game is as follows. The game graph itself is similar to the model, where decisions of the refuter correspond to the branching of the original model and decisions of the verifier correspond to the values given to undetermined propositions. The formula to be checked on the model is translated to the winning condition in the game. Much like the proofs of the lower bounds above, this straightforward translation may result in a game graph that is exponential in the number of propositions whose value is unknown. We now formalize these notions and prove that LTL and CTL generalized model checking is linearly reducible to decision of a game $G$. The formal exposition is more complicated than explained above as we ensure that the size of the game graph is linear in the number of unknown propositions.

A game is $G=\left\langle V, V_{1}, V_{2}, E, L, v, \varphi\right\rangle$ where $\langle V, E\rangle$ is a graph, $V_{1}$ and $V_{2}$ form a partition of $V$ to player 1 and player 2 vertices, respectively, $L: V \rightarrow 2^{A P}$ is a labeling of nodes with atomic propositions, $v \in V$ is an initial node, and $\varphi$ is a winning condition for player 1 given in either LTL or CTL over propositions AP. A strategy $\sigma$ for player 1 is $\sigma$ : $V^{*} \cdot V_{1} \rightarrow V$ such that for every $w \in V^{*}$ and $v_{1} \in V_{1}$ we have $\left(v_{1}, \sigma\left(w \cdot v_{1}\right)\right) \in E$. A strategy $\tau$ for player 2 is defined similarly. Given a strategy $\sigma$ for player 1 , the outcome of $\sigma$ from node $v$, denoted $\operatorname{out}(\sigma, v)$, is the tree $T \subseteq V^{*}$ such that for every $w \in V^{*}$ and $v_{1} \in V_{1}$ we have $\sigma\left(w \cdot v_{1}\right)$ is the unique son of $w \cdot v_{1}$ and for every $w \in V^{*}$ and $v_{2} \in V_{2}$ the nodes $\left\{w \cdot v_{2} \cdot v^{\prime} \mid\left(v_{2}, v^{\prime}\right) \in E\right\}$ is the set of sons of $w \cdot v_{2}$. Given a strategy $\sigma$ for player 1, a strategy $\tau$ for player 2, and a node $v$, the outcome of $\sigma$ and $\tau$ from $v$, denoted $\operatorname{out}(\sigma, \tau, v)$, is 
the word $w=v_{0} v_{1} \cdots \in V^{\omega}$ such that for every $i \geq 0$ we have if $v_{i} \in V_{1}$ then $v_{i+1}=\sigma\left(v_{0} \cdots v_{i}\right)$ and if $v_{i} \in V_{2}$ then $v_{i+1}=\tau\left(v_{0} \cdots v_{i}\right)$. Given a winning condition $\varphi$, a strategy $\sigma$ is winning for player 1 from node $v$ if $\varphi$ is in CTL and $\operatorname{out}(\sigma, v) \models \varphi$ and if $\varphi$ is in LTL and for every strategy $\tau$ for player 2 we have $\operatorname{out}(\sigma, \tau, v) \models \varphi$. Player 1 wins $G$ if she has a winning strategy from the initial node $v$. We decide a game $G$ by determining whether player 1 can win the game.

Theorem 16. LTL and CTL generalized model checking $M \models \preceq$ $\varphi$ is linearly reducible to decision of a game $G$.

Proof. Consider the PKS $M=\left\langle S, R, L, s^{i n}\right\rangle$ and the formula $\varphi$. Let $A P=\left\{p_{1}, \ldots, p_{n}\right\}$. Define the game $G=$ $\left\langle V, V_{1}, V_{2}, E, L^{\prime}, v^{i n}, \varphi^{\prime}\right\rangle$ as follows. Let $V^{\prime}=S \times[n] \times$ $\{$ true, false $\}$. Given a node $v=(s, i, \alpha) \in V^{\prime}$ we say that $v$ is consistent if either $\alpha=L\left(s, p_{i}\right)$ or $L\left(s, p_{i}\right)=\perp$. The set of nodes $V$ is $S \cup\left\{v \in V^{\prime} \mid v\right.$ is consistent $\}$ and $V_{1}=$ $S \cup(V \cap(S \times[n-1] \times\{$ true, false $\}))$ and $V_{2}=V \cap(S \times$ $\{n\} \times\{$ true, false $\})$. The set of edges $E$ is defined as follows. For a node $s \in V_{1}$ we have $(s,(s, 1, \beta)) \in E$ forall $\beta$ such that $(s, 1, \beta) \in V$. For a node $v=(s, i, \alpha) \in V_{1}$ we have $((s, i, \alpha),(s, i+1, \beta)) \in E$ forall $\beta$ such that $(s, i+1, \beta) \in$ $V$. For a node $v=(s, n, \alpha) \in V_{2}$ we have $\left((s, n, \alpha), s^{\prime}\right)$ forall $s^{\prime}$ such that $\left(s, s^{\prime}\right) \in R$. Finally, $L^{\prime}$ is defined over $A P^{\prime}=A P \cup\{q\}$ where $L^{\prime}$ is defined as follows.

$$
L^{\prime}(v, r)=\left\{\begin{array}{l}
\text { true If } r=p_{i} \text { and } v=(s, i, \text { true }) \\
\text { false If } r=p_{i} \text { and } v=(s, i, \text { false }) \\
\text { false If } r \neq p_{i} \text { and } v=(s, i, \alpha) \\
\text { true If } r=q \text { and } v \in S
\end{array}\right.
$$

Suppose that $\varphi$ is an LTL formula. Then, $\varphi^{\prime}$ is obtained from $\varphi$ by replacing (recursively) $p_{i}$ by $X^{i} p_{i}$, replacing $X \psi$ by $q \wedge X(\neg q U(q \wedge \psi))$, and $\psi_{1} U \psi_{2}$ by $\left(q \rightarrow \psi_{1}\right) U\left(q \wedge \psi_{2}\right)$.

Suppose that $\varphi$ is a CTL formula. Then $\varphi^{\prime}$ is obtained from $\varphi$ by replacing (recursively) $p_{i}$ by $E X^{i} p_{i}$, replacing $E X \psi$ by $q \wedge E X E(\neg q U(q \wedge \psi))$, replacing $A X \psi$ by $q \wedge$ $A X A(\neg q U(q \wedge \psi))$, replacing $E\left(\psi_{1} U \psi_{2}\right)$ by $E\left(\left(q \rightarrow \psi_{1}\right) U(q \wedge\right.$ $\left.\psi_{2}\right)$ ), and replacing $U\left(\psi_{1} U \psi_{2}\right)$ by $A\left(\left(q \rightarrow \psi_{1}\right) U\left(q \wedge \psi_{2}\right)\right)$.

In both cases it is possible to show that a winning strategy $\sigma$ for player 1 translates to a completion $M^{\prime}$ of $M$ such that $M^{\prime}$ satisfies $\varphi$ and that a completion $M^{\prime}$ of $M$ translates to a winning strategy for player 1 . It follows that player 1 wins iff GMC of $M$ with respect to $\varphi$ holds.

The number of nodes of $G$ can be further reduced to twice the number of states of $M$ by encoding all the propositions in $A P$ by one proposition $p$ in $G$ and augmenting $\varphi$ by clauses that force player 1 to leave cycles where $q$ does not hold.

\section{Conclusions}

We study generalized model checking for linear time properties. We consider the classical definition of GMC and show that it is 2EXPTIME-complete in the size of the formula and polynomial in the structure. We study a linear version of the completeness preorder and show that this preorder induces a
GMC problem that is EXPSPACE-complete in the size of the formula. We then proceed to show that for interesting classes of properties the model complexity can be restricted to a low order polynomial.

We have presented our work in the framework of partial Kripke structures. Other equally expressive 3-valued models [GJ03] include Modal Transition Systems [LT88] and Kripke Modal Transition Systems [HJS01]. All the complexity bounds given in this paper carry over to those closely related modeling formalisms.

We have seen that for interesting classes of LTL and CTL properties the complexity in term of the model can be restricted to linear or quadratic. We classify the properties according to deterministic word automata and nondeterministic tree automata that match these formulas. While most popular types of properties are covered above, characterization of the exact classes of formulas that can be translated to these types of automata is an interesting problem. That is, what are the exact subsets of LTL that can be translated to DWW and to DBW? Is there a simple syntactic way to express these subsets? The same problem for CTL (and other branching-time logics) involves tree automata. For every CTL property there exist an NBT and an AWT recognizing the same set of trees [KVW00]. What CTL properties can be translated to NWT? Is there a syntactic way to express these subsets? We know that if a word language can be recognized by a DBW and by a DCW, then it can be recognized by a DWW [KMM04]. This suggests the following natural question: Given a tree language that is accepted by an NCT and by an NBT, can it be recognized by an NWT? From a practical point of view, it could be interesting to study the specific case of CTL properties that are recognized by NCT.

Acknowledgements. We thank Michael Huth for comments on an earlier version and Orna Kupferman for a discussion of the relative expressive power of NBT and NCT.

\section{References}

$\left[\mathrm{AHL}^{+}\right.$08] A. Antonik, M. Huth, K. Larsen, U. Nyman, and A. Wasowski. 20 years of mixed and modal specifications. Bulletin of the European Association for Theoretical Computer Science, 2008.

[AMPS98] E. Asarin, O. Maler, A. Pnueli, and J. Sifakis. Controller synthesis for timed automata. In IFAC Symp. on System Structure and Control, pages 469-474. Elsevier, 1998.

[BG99] G. Bruns and P. Godefroid. Model checking partial state spaces with 3-valued temporal logics. In 11th Computer Aided Verification, pages 274-287, 1999.

[BG00] G. Bruns and P. Godefroid. Generalized model checking: Reasoning about partial state spaces. In 11th Concurrency Theory, volume 1877 of Lecture Notes in Computer Science, pages 168-182, 2000.

[BR01] T. Ball and S. Rajamani. The SLAM Toolkit. In 13th Computer Aided Verification, volume 2102 of Lecture Notes in Computer Science, pages 260-264, Paris, July 2001. Springer-Verlag. 
[GC05] A. Gurfinkel and M. Chechik. How Thorough is Thorough Enough? In 13th Correct Hardware Design and Verification Methods, 2005.

[GH05] P. Godefroid and M. Huth. Model Checking Vs. Generalized Model Checking: Semantic Minimizations for Temporal Logics. In 20th Logic in Computer Science, pages 158-167, Chicago, June 2005.

[GHJ01] P. Godefroid, M. Huth, and R. Jagadeesan. Abstractionbased Model Checking using Modal Transition Systems. In 12th Concurrency Theory, volume 2154 of Lecture Notes in Computer Science, pages 426-440, Aalborg, August 2001. Springer-Verlag.

[GJ02] P. Godefroid and R. Jagadeesan. Automatic Abstraction Using Generalized Model Checking. In 14th Computer Aided Verification, volume 2404 of Lecture Notes in Computer Science, pages 137-150. Springer-Verlag, 2002.

[GJ03] P. Godefroid and R. Jagadeesan. On the Expressiveness of 3-Valued Models. In 4th Verification, Model Checking and Abstract Interpretation, volume 2575 of Lecture Notes in Computer Science, pages 206-222, New York, January 2003. Springer-Verlag.

[GS97] S. Graf and H. Saidi. Construction of Abstract State Graphs with PVS. In 9th Computer Aided Verification, volume 1254 of Lecture Notes in Computer Science, pages 72-83, Haifa, June 1997. Springer-Verlag.

[GTW02] E. Grädel, W. Thomas, and T. Wilke. Automata, Logics, and Infinite Games. Lecture Notes in Computer Science 2500. Springer-Verlag, 2002.

[GWC06] A. Gurfinkel, O. Wei, and M. Chechik. Systematic Construction of Abstractions for Model-Checking. In 7th Verification, Model Checking, and Abstract Interpretation, volume 3855 of Lecture Notes in Computer Science, pages 381-397. Springer-Verlag, January 2006.

[HJMS02] T. Henzinger, R. Jhala, R. Majumdar, and G. Sutre. Lazy Abstraction. In 29th Principles of Programming Languages, pages 58-70, 2002.

[HJS01] M. Huth, R. Jagadeesan, and D. Schmidt. Modal Transition Systems: a Foundation for Three-Valued Program Analysis. In 10th European Symp. on Programming, volume 2028 of Lecture Notes in Computer Science. Springer-Verlag, 2001.

[Jur00] M. Jurdziński. Small progress measures for solving parity games. In 17th Theoretical Aspects of Computer Science, volume 1770 of Lecture Notes in Computer Science, pages 290-301. Springer-Verlag, 2000.

[Kle87] S. C. Kleene. Introduction to Metamathematics. North Holland, 1987.

[KMM04] O. Kupferman, G. Morgenstern, and A. Murano. Typeness for $\omega$-regular automata. In 2nd Automated Technology for Verification and Analysis, volume 3299 of Lecture Notes in Computer Science, pages 324-338. Springer-Verlag, 2004.

[KPP03] Y. Kesten, N. Piterman, and A. Pnueli. Bridging the gap between fair simulation and trace containment. In 15 th Computer Aided Verification, volume 2725 of Lecture Notes in Computer Science, pages 381-393. SpringerVerlag, 2003.

[KVW00] O. Kupferman, M.Y. Vardi, and P. Wolper. An automatatheoretic approach to branching-time model checking. Journal of the ACM, 47(2):312-360, 2000.

[LT88] K. G. Larsen and B. Thomsen. A Modal Process Logic. In 3rd Logic in Computer Science, pages 203-210, 1988.
[MP90] Z. Manna and A. Pnueli. A hierarchy of temporal properties. In 9th Symposium on Principles of Distributed Computing, pages 377-410. ACM, 1990.

[MP92] Z. Manna and A. Pnueli. The Temporal Logic of Reactive and Concurrent Systems: Specification. Springer-Verlag, Berlin, January 1992.

[Pit07] N. Piterman. From nondeterministic Büchi and Streett automata to deterministic parity automata. Logical Methods in Computer Science, 3(3):5, 2007.

[PPS06] N. Piterman, A. Pnueli, and Y. Saar. Synthesis of reactive(1) designs. In 7th Verification, Model Checking, and Abstract Interpretation, volume 3855 of Lecture Notes in Computer Science, pages 364-380. Springer-Verlag, 2006.

[PR89] A. Pnueli and R. Rosner. On the synthesis of a reactive module. In 16th Principles of Programming Languages, pages 179-190, 1989.

[RW89] P.J.G. Ramadge and W.M. Wonham. The control of discrete event systems. IEEE Transactions on Control Theory, 77:81-98, 1989.

[Saf88] S. Safra. On the complexity of $\omega$-automata. In 29th Foundations of Computer Science, pages 319-327, White Plains, October 1988.

[SVW87] A.P. Sistla, M.Y. Vardi, and P. Wolper. The complementation problem for Büchi automata with applications to temporal logic. Theoretical Computer Science, 49:217237, 1987.

[vEB97] P. van Emde Boas. The convenience of tilings. In Complexity, Logic and Recursion Theory, volume 187 of Lecture Notes in Pure and Applied Mathetaics, pages 331363, 1997.

[VW86] M.Y. Vardi and P. Wolper. Automata-theoretic techniques for modal logics of programs. Journal of Computer and System Science, 32(2):182-221, April 1986.

[VW94] M.Y. Vardi and P. Wolper. Reasoning about infinite computations. Information and Computation, 115(1):1-37, November 1994. 